\title{
Final state interactions and hadron quenching in cold nuclear matter
}

\author{
A. Accardi \\ Dept. of Physics and Astronomy, Iowa State U., Ames, IA 50011, USA
}

\begin{abstract}
I examine the role of final state interactions in cold nuclear matter in modifying hadron production on nuclear targets with leptonic or hadronic beams. I demonstrate the extent to which available experimental data in electron-nucleus collisions can give direct information on final state effects in hadron-nucleus and nucleus-nucleus collisions. For hadron-nucleus collisions, a theoretical estimate based on a parton energy loss model tested in lepton-nucleus collisions shows a large effect on mid-rapidity hadrons at fixed target experiments. At RHIC energy, the effect is large for negative rapidity hadrons, but mild at midrapidity. This final state cold hadron quenching needs to be taken into account in jet tomographic analysis of the medium created in nucleus-nucleus collisions.
\end{abstract}

\section{INTRODUCTION}

Hadron production on nuclear targets is strongly influenced by the presence of cold and hot nuclear matter. The most spectacular effects is jet quenching in nucleusnucleus $(A+A)$ collisions [1-4], namely, the suppression of hadron production at large transverse momentum compared to a suitably scaled cross section in protonproton collisions. This phenomenon is widely used as a tool to explore the properties of the hot QCD medium produced in heavy-ion collisions [5-7], and as evidence for the creation of a novel state of matter at the Relativistic Heavy Ion Collider (RHIC) [1-4], possibly the Quark-Gluon Plasma (QGP) [8]. Hadron suppression has also been observed in lepton-nucleus $(\ell+A)$ [9-14] and hadron-nucleus $(h+A)$ collisions [15-17], where one does not expect the formation of an extended hot medium. In this case, the target nucleus itself (cold nuclear matter) induces the observed suppression of hadron production, which I will refer to as "cold hadron quenching", or cold quenching in short.

Nuclear effects in cold nuclear matter can be classified as initial-state or final-state depending on whether they happen before or after the hard collision which generates the hard probe. Final state (FS) effects can be isolated in semi-inclusive hadron production in $\ell+A$ collisions. Hadron suppression in these collisions [9-13] is typically attributed to radiative energy loss of the struck quark or to nuclear absorption of a colorless prehadron, see [18] and references therein. Initial state (IS) effects can be experimentally isolated in Drell-Yan processes in $h+A$ collisions [19-21], and they are attributed to nuclear shadowing or radiative energy loss of the incoming parton [22-24]. In large- $p_{T}$ hadron production in $h+A$ collisions both IS and FS effects are present, and they cannot be easily disentangled. They give rise to a host of interesting effects. They modify the shape of midrapidity hadron $p_{T}$ spectra, slightly suppressing it at small $p_{T} \lesssim 1-2$ $\mathrm{GeV}$ and enhancing it at intermediate $2 \mathrm{GeV} \lesssim p_{T} \lesssim 6$ $\mathrm{GeV}$; this is known as Cronin effect [25-27]. An extensive study of the rapidity dependence of the Cronin effect in deuteron-gold $(d+A u)$ collisions at RHIC has shown an interesting evolution of the Cronin effect: the Cronin enhancement grows at backward rapidity $y-y_{c m}<0$
[28-31]; however, at forward rapidity $y-y_{c m}>0$, the spectrum is suppressed up to large $p_{T}$ [15-17], a trend confirmed also at lower energy collisions [32, 33]. The forward rapidity suppression has been widely interpreted as evidence for the onset of the Color Glass Condensate, a universal state of highly saturated quark and gluons in the nuclear wave function $[34,35]$. However, explanations in terms of IS energy loss and higher-twist shadowing [33], leading-twist shadowing [36], Sudakov suppression [37] or FS parton recombination [38] have been equally successful in describing the data. The rise of the Cronin effect at backward rapidity is more difficult to understand [39]. Explanations in terms of IS anti-shadowing [40] or saturation [28] have been proposed. Finally, recent PHENIX data on neutral pion production in $d+A u$ collisions at midrapidity suggest a small suppression of midrapidty $\pi^{0}$ at $p_{T} \gtrsim 10 \mathrm{GeV}$ [41]. It cannot be explained by the EMC effect, which is effective at $p_{T} \gtrsim 15$ $\mathrm{GeV}[42,43]$, but may accommodate a small final state energy loss of order $10 \%$ [43].

A consistent interpretation of this wealth of experimental data requires a deep understanding of IS and FS interactions at the parton and hadron level, and the development of a unified computational framework [44]. As a contribution to this program, in this paper I will analyze phenomenologically the contribution of final state interactions to hadron production in $h+A$ and $A+A$ collisions, and I will show that it is indeed non negligible in the whole backward rapidity hemisphere up to RHIC energy. At the Large Hadron Collider (LHC) it will be important only at very backward rapidity $y-y_{c m} \lesssim 3$. In Section II, I will review the kinematics of hadron production at leading order (LO) in perturbative QCD for Deep Inelastic Scattering (DIS) and nucleon-nucleon collisions $(\mathrm{NN})$, and build a dictionary that relates the kinematic variables used in the 2 cases. In Section III, I will compare the NN and DIS phase spaces at present and future experiments in terms of either set of variables, to understand in detail the relevance of NN to DIS and viceversa. In Section IV, I will use the developed kinematic dictionary to show the extent to which present $\ell+A$ experimental data can give information on final state cold nuclear matter effects in $h+A$ and $A+A$ collisions (collectively, $A+B$ collisions). Then, I will use an energy 

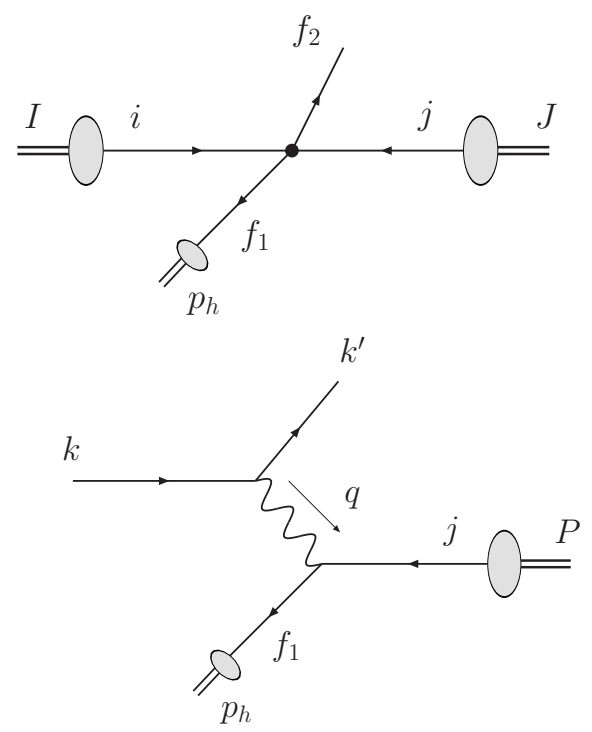

FIG. 1: Left: LO kinematics for parton production in NN collisions - double lines indicate hadrons or nuclei, single lines are partons. Right: LO kinematics for parton production in DIS collisions - double lines indicate hadrons or nuclei, thin single lines are partons (bottom) or leptons (top). The labels define the particles 4-momenta.

loss model tuned to $\ell+A$ data to estimate cold quenching in $h+A$ collisions for midrapidity hadrons at the Super Proton Synchrotron (SPS) and at Fermilab (FNAL), and at various rapidities at RHIC. A comparison of FS and IS effects will be discussed in Section V, and my conclusions reported in Section VI.

\section{PARTON PRODUCTION IN DIS AND NN COLLISIONS}

Considering parton and hadron production at LO in $\mathrm{NN}$ collisions and DIS collisions, it is easy to provide an explicit dictionary translating between the variables traditionally used in the analysis of the 2 processes. I will start by discussing the kinematics of parton and hadron production in NN collisions in the center-of-mass frame (c.m.f.). I will then suitably identify the DIS kinematics in terms of $\mathrm{NN}$ variables, and derive the dictionary. I will work in the framework of collinear factorization in pQCD, and use light-cone coordinates throughout this discussion: for any 4-vector $a^{\mu}$ I write $a^{\mu}=\left(a^{+}, a^{-}, \vec{a}_{T}\right)$, where $a^{ \pm}=\left(a^{0} \pm a^{3}\right) / \sqrt{2}$ are the plus- and minus-momenta and $\vec{a}_{T}=\left(a^{1}, a^{2}\right)$ the transverse momentum.

\section{A. NN collisions}

In $\mathrm{pQCD}$ at leading order in the coupling constant $\alpha_{s}$, parton production in NN collisions proceeds through $2 \rightarrow 2$ partonic collisions (see Fig. 1 left and Table II for the definition of kinematic variables.) Several LO processes can contribute to a given $i j \rightarrow f_{1} f_{2}$ collisions, represented by a black disk in the cartoon, see Ref [45] for details. The momenta of the 2 nucleons colliding in the c.m.f. with energy $\sqrt{s} / 2$ each are

$$
\begin{aligned}
& I=\left(\sqrt{\frac{\tilde{s}}{2}}, \frac{M^{2}}{\sqrt{2 \tilde{s}}}, \overrightarrow{0}_{T}\right) \\
& J=\left(\frac{M^{2}}{\sqrt{2 \tilde{s}}}, \sqrt{\frac{\tilde{s}}{2}}, \overrightarrow{0}_{T}\right)
\end{aligned}
$$

where $M$ is the nucleon mass and

$$
\tilde{s}=s \frac{1+\sqrt{1+M^{4} / s^{2}}}{2} .
$$

I will neglect terms of order $O\left(M^{2} / s\right)$ compared to terms of $O(1)$, and will use $\tilde{s} \approx s$. Note also that in the definition of the nucleon momenta, I explicitly retain the nucleon mass in Eq. (1) to be able to perform boosts to the rest frame of either nucleon. If we assume the partons to be massless and collinear to their parent nucleons, their 4 -momenta in terms of the parton fractional momenta $x_{i}$ read

$$
\begin{aligned}
& i=\left(x_{1} \frac{\sqrt{s}}{2}, 0, \overrightarrow{0}_{T}\right) \\
& j=\left(0, x_{2} \frac{\sqrt{s}}{2}, \overrightarrow{0}_{T}\right) .
\end{aligned}
$$

In terms of rapidities and transverse momentum $p_{T}$, the parton 4-momenta read

$$
\begin{aligned}
& f_{1}=\left(\frac{p_{T}}{\sqrt{2}} e^{y_{1}}, \frac{p_{T}}{\sqrt{2}} e^{-y_{1}},-\vec{p}_{T}\right) \\
& f_{2}=\left(\frac{p_{T}}{\sqrt{2}} e^{y_{2}}, \frac{p_{T}}{\sqrt{2}} e^{-y_{2}}, \vec{p}_{T}\right) .
\end{aligned}
$$

We can express the parton fractional momenta in terms of $p_{T}, y_{i}$ as

$$
\begin{aligned}
& x_{1}=\frac{p_{T}}{\sqrt{s}}\left(e^{y_{1}}+e^{y_{2}}\right) \\
& x_{2}=\frac{p_{T}}{\sqrt{s}}\left(e^{-y_{1}}+e^{-y_{2}}\right) .
\end{aligned}
$$

Finally, the Mandelstam invariants are defined as follows,

$$
\begin{aligned}
& \hat{s}=(i+j)^{2} \\
& \hat{t}=\left(i-f_{1}\right)^{2}=\left(f_{2}-j\right)^{2} \\
& \hat{u}=\left(i-f_{2}\right)^{2}=\left(f_{1}-j\right)^{2}
\end{aligned}
$$

and 4-momentum conservation is expressed as $\hat{s}+\hat{t}+\hat{u}=$ 0 . In terms of rapidities and transverse momentum, the Mandelstam invariants read

$$
\begin{aligned}
& \hat{s}=x_{1} x_{2} s \\
& \hat{t}=-p_{T}^{2}\left(1+e^{y_{2}-y_{1}}\right) \\
& \hat{u}=-p_{T}^{2}\left(1+e^{y_{1}-y_{2}}\right) .
\end{aligned}
$$




\begin{tabular}{rlrl}
\hline Variable & Definition & & \\
\hline $\boldsymbol{s}$ & & Nucleon-nucleon center of mass energy squared. \\
$x_{1}=i^{+} / I^{+}$ & & Initial state projectile parton fractional momentum. \\
$x_{2}$ & $=j^{-} / J^{-}$ & & Initial state target parton fractional momentum. \\
$\vec{p}_{i T}$ & $=\left|\vec{f}_{i T}\right|$ & & Final state partons transverse momentum (relative to beam). \\
$y_{i}$ & $=0.5 \log \left(f_{i}^{+} / f_{i}^{-}\right)$ & Final state partons rapidity. \\
$y_{c m}$ & $=0.5 \log \left(\frac{I^{+}+J^{+}}{I^{-}+J^{-}}\right)$ & & Rapidity of the center of mass. \\
\hline$z$ & $=p_{h}^{+} / f_{1}^{+}$ & & Hadron fractional momentum relative to parent parton $f_{1}$. \\
$\boldsymbol{p}_{\boldsymbol{h} \boldsymbol{T}}$ & $=\left|\vec{p}_{h T}\right|$ & & Hadron transverse momentum (relative to beam). \\
$\boldsymbol{y}_{\boldsymbol{h}}$ & $=0.5 \log \left(p_{h}^{+} / p_{h}^{-}\right)$ & Hadron rapidity. \\
$\boldsymbol{\eta}$ & $=-\log \tan \left(\theta^{*} / 2\right)$ & & Hadron pseudorapidity $\left(\theta^{*}\right.$ is the angle between the parton \\
& & & and the beam in the center of mass reference frame). \\
\hline
\end{tabular}

TABLE I: Definitions of the kinematic variables for semi-inclusive parton and hadron production in pQCD (top and bottom part of the table, respectively). Particle 4-momenta are defined in Fig. 1. Boldface variables are experimentally measurable. The remaining variables are theoretically defined in the QCD parton model in collinear factorization. Note that at LO, with 2 final state partons, $\vec{p}_{1 T}=-\vec{p}_{2 T}=\vec{p}_{T}$.

\begin{tabular}{|c|c|c|}
\hline Tariable Definition & Target rest frame & \\
\hline$M^{2}=P^{2}$ & & Target mass. \\
\hline $\boldsymbol{x}_{B}=\frac{-q^{2}}{O P}$ & & Bjorken scaling variable. \\
\hline$Q^{2}=-q^{2}$ & & Negative four-momentum squared of the virtual photon. \\
\hline$\nu=\frac{q \cdot P}{\sqrt{P^{2}}}$ & $=E_{e}^{t r f}-E_{e}^{t r f \prime}$ & Energy of the virtual photon in the target rest frame. \\
\hline $\boldsymbol{y}=\frac{q \cdot P}{k \cdot P}$ & $=\frac{\nu}{E^{t r f}}$ & Fractional energy loss of the incident lepton. \\
\hline $\boldsymbol{W}^{\mathbf{2}}=(P+q)^{2}$ & & Invariant mass squared of the hadronic final state. \\
\hline$z_{h}=\frac{p_{h} \cdot P}{q \cdot P}$ & $=\frac{E_{h}}{\nu}$ & Fraction of the virtual photon energy carried by the hadron. \\
\hline $\boldsymbol{p}_{\boldsymbol{T}}=\left|\vec{p}_{T}\right|$ & & Hadron transverse momentum (relative to the virtual photon momentum). \\
\hline
\end{tabular}

TABLE II: Definitions of the kinematic variables for semi-inclusive DIS. The Lorentz invariant definition and its form in the target rest frame are provided. Particle 4-momenta are defined in Fig. 1. All variables are experimentally measurable, hence typeset in boldface. Note that $x_{B}=Q^{2} /(2 M \nu)$ independently of the chosen reference frame.

In order to compare collider and fixed target experiments, and different beam energies, it is useful to consider the rapidity in the c.m.f.:

$$
y_{c . m . f .}=y-y_{c m} .
$$

The backward rapidity region (target hemisphere) corresponds to $y-y_{c m}<0$, and the forward rapidity region (projectile hemisphere) to $y-y_{c m}>0$.

Hadronization in the collinear factorization framework proceeds through independent parton fragmentation into a hadron. It is universal, i.e., independent of the process which produced the fragmenting hadron, e.g., NN or DIS collisions [46]. The hadron fractional momentum $z$ is defined by

$$
\begin{gathered}
p_{h}^{+}=z f_{1}^{+} \\
\vec{p}_{h T}=z \vec{f}_{1 T} .
\end{gathered}
$$

Therefore the on-shell hadron momentum $p_{h}$ reads

$$
p_{h}=\left(z f_{1}^{+}, \frac{m_{h}^{2}+z^{2} f_{1 T}^{2}}{2 z f_{1}^{+}}, z \vec{f}_{1 T}\right) \text {. }
$$

The parton and hadron rapidities are related by $y_{1}=$ $y_{h}+\log \left(m_{h T} / p_{h T}\right)$. The non perturbative dynamics of the fragmentation process is encoded in universal fragmentation functions, which can be obtained in global fits of experimental data $[47,48]$.

\section{B. DIS collisions}

At LO in pQCD, deeply inelastic scattering proceeds by exchange of a virtual photon in the $\hat{t}$-channel, explicitly shown in Fig. 1 right. The DIS Lorentz invariants are defined in Table II. Semi-inclusive nDIS is best discussed in terms of $\nu$ and $Q^{2}$, which are the most relevant variables to hadron quenching processes in nuclear targets. Analysis of inclusive DIS is usually carried out using $x_{B}$ and $Q^{2}$.

DIS experiments can be performed with a fixed target $(\mathrm{ft})$ or in collider mode $(\mathrm{cl})$. Examples are the EMC, HERMES, JLAB experiments, and the Electron-Ion Collider (EIC), respectively. The colliding nucleon and lepton momenta are

$$
\begin{aligned}
P_{f t} & =\left(\frac{M}{\sqrt{2}}, \frac{M}{\sqrt{2}}, \overrightarrow{0}_{T}\right), \quad k_{f t}=\left(\sqrt{2} E_{e}, 0, \overrightarrow{0}_{T}\right) \\
P_{c l} & =\left(\frac{M}{2 \sqrt{2} E_{N}}, \sqrt{2} E_{N}, \overrightarrow{0}_{T}\right), \quad k_{c l}=\left(\sqrt{2} E_{e}, 0, \overrightarrow{0}_{T}\right)
\end{aligned}
$$

where $E_{e}$ and $E_{N}$ are the electron and nucleon energies measured in the laboratory frame. To discuss both modes at the same time, it is convenient to introduce the target 
rest frame energy of the electron, $E_{e}^{\text {trf: }}$

$$
E_{e}^{t r f}= \begin{cases}E_{e} & \text { fixed target } \\ \frac{2 E_{N} E_{e}}{M} & \text { collider mode }\end{cases}
$$

Then the invariant $y$ for both modes becomes $y=\nu / E_{e}^{\text {trf }}$.

\section{The dictionary}

To connect the DIS and NN kinematics, we can boost the DIS collision to a frame in which the target has energy $\sqrt{s} / 2$ per nucleon. Then, we can imagine the lepton to be a parton of a phantom nucleon of energy $\sqrt{s} / 2$ collinear with the lepton, with 4-momentum $P^{\prime \pm}=P^{\mp}$. Comparing the top and bottom of Fig.1 we can identify

$$
P \equiv J, \quad P^{\prime} \equiv I, \quad k \equiv i, \quad k^{\prime} \equiv f_{2} .
$$

The virtual photon momentum $q$, the fractional momentum $x_{e}$ of the initial state lepton and the rapidity $y_{e}$ of the final state lepton are identified as follows

$$
q=k-k^{\prime} \equiv i-f_{2}, \quad x_{e}=k^{+} / P^{\prime+} \equiv x_{1}, \quad y_{e} \equiv y_{2} .
$$

In this way, we can relate the DIS kinematics to the NN kinematics discussed in Sect. II A. As an example, it is immediate to see that, in terms of NN variables, $Q^{2}=-\hat{t}$. The full translation dictionary from DIS to NN variables can be obtained in a straightforward way by combining the results of Sects. II A-II B and the definitions of Tables I-II.

First, we can express the DIS invariants in terms of parton rapidities and transverse momenta. Neglecting target mass corrections, i.e., up to terms of $O\left(M^{2} / s\right)$, we obtain

$$
\begin{aligned}
x_{B} & =\frac{p_{T}}{\sqrt{s}}\left(e^{-y_{2}}+e^{-y_{1}}\right) \\
Q^{2} & =p_{T}^{2}\left(1+e^{y_{1}-y_{2}}\right) \\
\nu & =\frac{p_{T} \sqrt{s}}{2 M} e^{y_{1}} \\
y & =\frac{1}{1+e^{y_{2}-y_{1}}} \\
z_{h} & =z .
\end{aligned}
$$

Note that the first 3 variables are not independent because $Q^{2}=2 M x_{B} \nu$, and that $x_{B}=x_{2}$ is interpreted as the struck parton fractional momentum, as expected in DIS at LO. Note also that $\nu$ increases with increasing $p_{T}$ and increasing $y_{1}$. In other words, a parton of positive and large $y_{1}$ travels in the opposite direction as its parent nucleon, hence in the target rest frame it is very fast. Conversely, a parton of negative and large $y_{1}$ travels in the same direction as its parent nucleon, which means quite slow in the target rest frame. It is also interesting to note that up to terms of order $O\left(M^{2} / s\right)$, the parton and hadron energy in the target rest frame are

$$
E=\nu \quad E_{h}=z_{h} \nu .
$$

Finally, we can invert Eq. (15) to obtain the NN variables in terms of DIS invariants:

$$
\begin{aligned}
p_{T}^{2} & =(1-y) Q^{2} \\
y_{1} & =-\log \left(\frac{Q \sqrt{s}}{2 M E_{e}^{t r f}} \frac{(1-y)^{1 / 2}}{y}\right) \\
y_{2} & =y_{1}+\log \left(\frac{1-y}{y}\right) \\
z & =z_{h}
\end{aligned}
$$

with $y=\nu / E_{e}^{\text {trf }}$.

Note that in DIS, the electron energy $E_{e}^{\text {trf }}$, hence the electron $x_{e}$, is fixed by the experimental conditions; this is different from $\mathrm{NN}$ collisions where the parton $j$ has an unconstrained fractional momentum. Changing the c.m.f. energy to $\sqrt{s^{\prime}}$ simply results in a shift of the parton rapidity,

$$
y_{1} \underset{s \rightarrow s^{\prime}}{\longrightarrow} y_{1}+\Delta y_{1}
$$

where $\Delta y_{1}=\log \left(\sqrt{s} / \sqrt{s^{\prime}}\right)$. The value of $\Delta y_{1}$ compared to RHIC top energy $\sqrt{s}=200 \mathrm{GeV}$ is listed in Table III for the experiments of interest in this paper. Another difference between DIS and NN collisions is the rapidity difference $\Delta y$ between the outgoing "partons". In DIS, the electron fractional momentum is fixed, so that $\Delta y_{\mid D I S}=y_{1}-y_{e}=\log (y /(1-y))$ is determined for each $p_{T}$ and $y_{1}$ by the corresponding value of $y=\nu / E_{e}^{\text {trf }}$, and can span only a limited range:

$$
\log \left(\frac{y_{\min }}{1-y_{\min }}\right) \leq \Delta y_{\mid D I S} \leq \log \left(\frac{y_{\max }}{1-y_{\max }}\right) .
$$

For example, at HERMES the experimental acceptance $0.07<y<0.85$ translates into $-1.1<\Delta y_{\mid D I S}<0.75$. In $\mathrm{NN}$ collisions, neither parton fractional momentum is fixed by the experimental conditions, hence $\Delta y_{N N}=$ $y_{1}-y_{2}$ can span

$$
-\log \left(\frac{\sqrt{s} e^{-y_{1}}}{p_{T}}-1\right) \leq \Delta y_{\mid N N} \leq \log \left(\frac{\sqrt{s} e^{y_{1}}}{p_{T}}-1\right) .
$$

For example, for an observed parton with $y_{1}=-2$ and $p_{T}=2 \mathrm{GeV}$, corresponding to the middle of the HERMES DIS phase space, we obtain $-2.5<\Delta y_{\mid N N}<6.6$, even though the average $\left\langle\Delta y_{\mid N N}\right\rangle$ will lay in the middle of this interval.

\section{COMPARING THE PHASE SPACES}

We can now compare in detail the phase spaces for parton production in NN and DIS collisions. For this purpose, I will define a NN-equivalent DIS phase space and a DIS-equivalent NN phase space. 

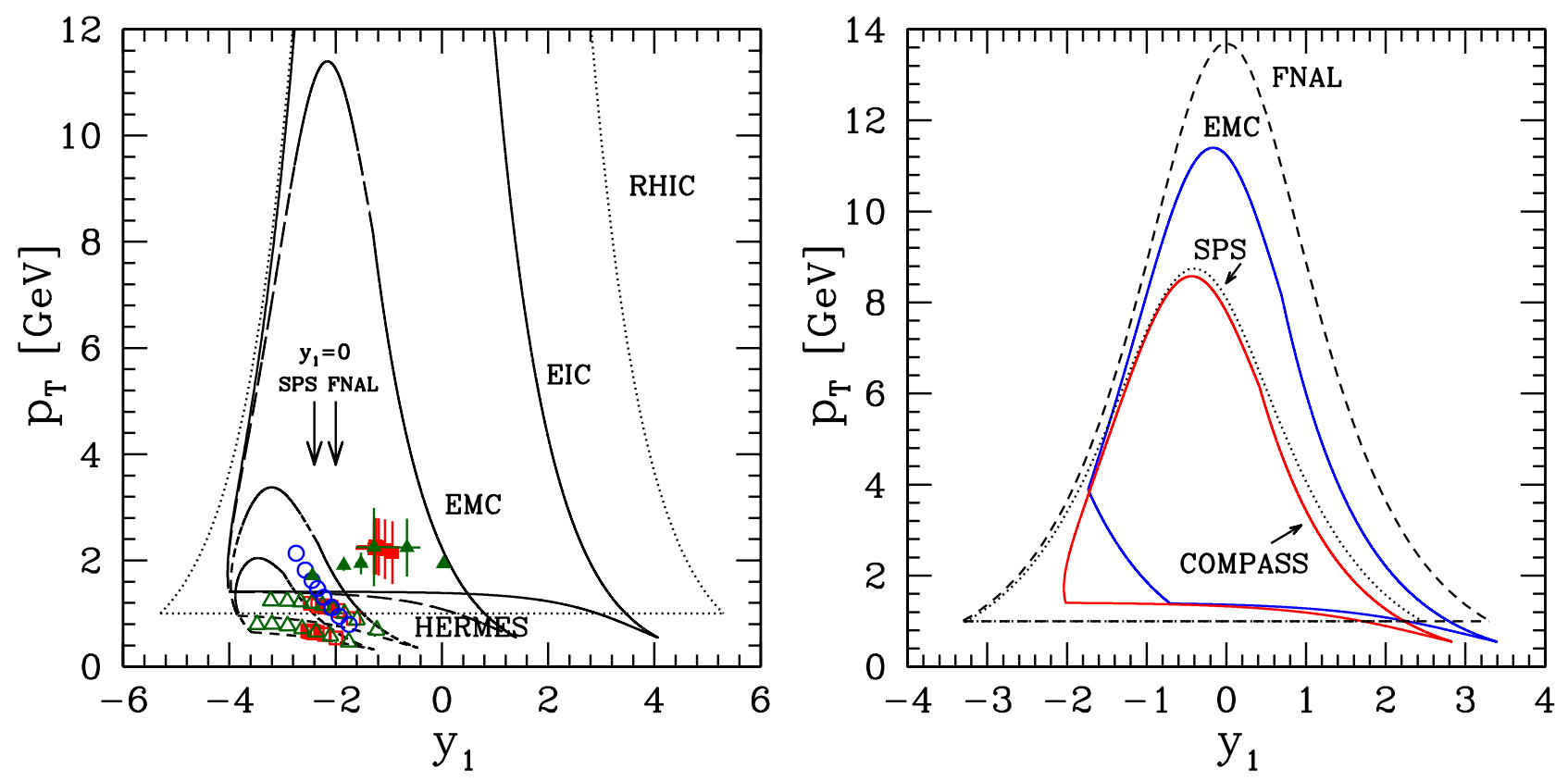

FIG. 2: Left: the RHIC-equivalent phase space of DIS experiments at $E_{e}=27.6 \mathrm{GeV}$ (HERMES, solid line), at $E_{e}=12 \mathrm{GeV}$ (HERMES and JLAB, dashed line), and at $E_{e}=280 \mathrm{GeV}$ (EMC, dot-dashed line). The dotted line shows the borders of the LO pQCD phase space at top RHIC energy, $\sqrt{s}=200 \mathrm{GeV}$. The 2 arrows show the location of the midrapidity region at SPS and FNAL fixed target experiments. The open and filled symbols show the position of a representative selection of data on hadron suppression collected at the HERMES [13, 49] and EMC experiments [10], respectively. Red squares are for $z_{h}$-distributions, green triangles for $\nu$-distributions and blue circles for $Q^{2}$-distributions. The systematic uncertainties on EMC data, shown as error bars, are discussed in Section IV A. Right: NN-equivalent EMC and COMPASS phase space at $\sqrt{s}=27.4$ $\mathrm{GeV}$, compared to the SPS and FNAL phase spaces.

\begin{tabular}{|c|c|c|c|c|c|}
\hline & & 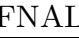 & & & \\
\hline$\sqrt{s}[\mathrm{GeV}$ & 17.5 & 27.4 & 63 & 200 & 50 \\
\hline$\Delta y_{1}$ & 2.4 & 2.0 & 1.2 & 0 & -3 \\
\hline
\end{tabular}

TABLE III: Rapidity shifts $\Delta y_{1}$ of the RHIC-equivalent DIS phase space, tabulated for some energies of interest.

\section{A. NN-equivalent DIS phase space}

Given a DIS phase space, i.e., a given experiment acceptance region in the $\left(\nu, Q^{2}\right)$ plane, I define its $N N$ equivalent phase space as its image in the $\left(p_{T}, y_{1}\right)$ under Eqs. (17). (I do not consider the transformation of the fragmentation variable $z_{h}$ into $z$ because of its triviality.) The reason for this definition is that for both NN and DIS collisions we can identify the parton $f_{1}$ of Fig. 1 with the "observed" parton in NN and DIS collisions, i.e., the parton which fragments into the observed hadron. Then the variables $p_{T}$ and $y_{1}$ fully characterize the observed parton. An analogous definition holds when using $x_{B}$ instead of $\nu$ as independent variable.

As an example, the HERMES DIS phase space in the $\left(\nu, Q^{2}\right)$ plane is determined by the values of $W_{\text {min }}^{2}, Q_{\text {min }}^{2}$ and $y_{\max }$ :

$$
\begin{aligned}
& \frac{Q_{\min }^{2}+W_{\min }^{2}-M^{2}}{2 M} \leq \nu \leq y_{\max } E_{e}^{t r f} \\
& Q_{\min }^{2} \leq Q^{2} \leq M^{2}+2 M \nu-W_{\min }^{2}
\end{aligned}
$$

Additionally, one may impose stronger cuts on $\nu$, e.g., $\nu \geq \nu_{\min }$, as at the EMC experiment, and in some HERMES analysis.

With Eqs. (17) it is easy to plot the NN-equivalent DIS phase space in the $\left(y_{1}, p_{T}\right)$ plane. As an example, we can consider the RHIC-equivalent phase space of the HERMES and EMC experiments, using $\sqrt{s}=200 \mathrm{GeV}$, shown in Fig. 2 left. Note that according to Eq. (18), the NN-equivalent phase space at other center of mass energies can be obtained by a shift $y_{1} \rightarrow y_{1}+\Delta y_{1}$, see Table III. I assume the pQCD formulae used to define the NN-equivalent phase space to be valid at RHIC top energy for $p>p_{0}=1 \mathrm{GeV}$ : the corresponding pQCD confidence region is plotted as a dotted line, see Eq. (23) for details.

We can see that the HERMES experiment, with $E_{e}^{\text {trf }}=$ 12 and $27.6 \mathrm{GeV}$, covers less than one third of the available RHIC $p_{T}$ range at $y_{1} \approx-3$, with shrinking $p_{T}$ coverage at larger rapidity. In the SPS/FNAL midrapidity 
region it reaches $p_{T}=2.5 \mathrm{GeV}$ at most. Since

$$
y_{1} \leq \log \left(\frac{\sqrt{s}}{2 M E_{e}^{t r f}} \frac{p_{T}}{y_{\max }}\right)
$$

and $y_{\max }$ cannot be increased above 1 , the only way to effectively reach larger values of $y_{1}$ is to increase the electron beam energy $E_{e}^{t r f}$. Indeed, the EMC experiment, with $E_{e}^{\text {trf }}=100-280 \mathrm{GeV}$, covers a larger span in rapidity and extends to $y_{1} \gtrsim 0$. Moreover, the increased energy allows in principle to reach much higher $p_{T}$ than at HERMES. However, only the $p_{T} \lesssim 3 \mathrm{GeV}$ region has been explored. As also shown in Fig. 2 left, the proposed Electron-Ion Collider (EIC) $[50,51]$ will be able to effectively study the $y_{1}>0$ region, and cover most of the RHIC phase space. Likewise, it will cover only the $y_{1}<0$ part of the LHC phase space.

The reason why present experimental data in $\ell+A$ collisions reach only $p_{T} \lesssim 2 \mathrm{GeV}$, is that conventional DIS variables $z_{h}, \nu$ or $Q^{2}$ explore the available $\mathrm{NN}$-equivalent phase space in an uneven way. Moreover, in single differential distributions like $d N_{A}^{h} / d z$, the integration over the remaining variables favors low values of $Q^{2}$, hence low$p_{T}$ values. While HERMES is inherently limited in its $p_{T}$ reach by the low electron beam energy, the EMC experiment covers, in principle, most of the SPS and FNAL phase space, see Fig. 2 right. Therefore, a rebinning of the EMC experimental data in terms of $\mathrm{NN}$ variables would result in an experimental measurements of final state nuclear effects, much needed for correctly interpreting large- $p_{T}$ hadron spectra in $h+A$ and $A+A$ collisions at SPS and FNAL. Another possibility would be to study $\mu+A$ collisions at the COMPASS experiment [52], which has a muon beam energy of $E_{\mu}=160 \mathrm{GeV}$ comparable to EMC, and whose phase space is also shown in the plot.

\section{B. DIS-equivalent NN phase space}

When discussing $\mathrm{NN}$ collisions in the framework of collinear factorization in $\mathrm{pQCD}$, we should first define the region of validity of perturbative computations: $p_{T} \geq p_{0}$. Typically one needs $p_{0} \gtrsim 1 \mathrm{GeV}$, which agrees with the phenomenological analysis of Refs. [42, 53]. Then, the $\mathrm{NN}$ phase space at a given $y_{1}$ is defined by the kinematic bounds on $2 \rightarrow 2$ parton scatterings [42]:

$$
\begin{aligned}
& \left|y_{1}\right| \leq \cosh ^{-1}\left(\frac{\sqrt{s}}{2 p_{0}}\right) \\
& p_{0} \leq p_{T} \leq \frac{\sqrt{s}}{2 \cosh \left(y_{1}\right)} \\
& -\log \left(\frac{\sqrt{s}}{p_{T}}-e^{-y_{1}}\right) \leq y_{2} \leq \log \left(\frac{\sqrt{s}}{p_{T}}-e^{y_{1}}\right) \\
& \frac{m_{h T}}{\sqrt{s}} e^{y_{h}}\left(1+\frac{p_{h T}^{2}}{m_{h T}^{2} e^{y_{h}}}\right) \leq z \leq 1
\end{aligned}
$$

Introduction of intrinsic parton transverse momentum in the formalism, or use of next-to-leading order kinematics
[54], would relax somewhat these bounds. We should also keep in mind that at large rapidity, where the $2 \rightarrow 2$ phase space is becoming more and more restricted, $2 \rightarrow 1$ parton fusion processes may become the dominant mechanism, because they are sensitive to much lower fractional momenta $x_{i}$ [39]. Hence, at the boundary of the NN phase space, the presented analysis becomes unreliable.

The DIS-equivalent NN phase space is defined as the image of Eqs. (23) in the $\left(\nu, Q^{2}, y, z_{h}\right)$ space under Eqs. (15). It is 4-dimensional and difficult to directly visualize. A way around this problem is to define suitable trajectories in NN phase space averaged over $y_{2}$, and to project them into the DIS-equivalent $\left(\nu, Q^{2}\right)$ and $\left(\nu, z_{h}\right)$ phase spaces. We can define a $p_{h T^{-}}$and $y_{h}$-dependent average observable as follows

$$
\langle\mathcal{O}\rangle_{p_{h T}, y_{h}}=\frac{\int d z d y_{1} d y_{2} \mathcal{O}\left(p_{T}, y_{1}, y_{2}, z\right) \frac{d \hat{\sigma}^{A B \rightarrow h X}}{d p_{T}^{2} d y_{1} d y_{2} d z}}{\int d z d y_{1} d y_{2} \frac{d \hat{\sigma}^{A B \rightarrow h X}}{d p_{T}^{2} d y_{1} d y_{2} d_{z}}},
$$

where

$$
\frac{d \hat{\sigma}^{A B \rightarrow h X}}{d p_{T}^{2} d y_{1} d y_{2} d z}=\sum_{f_{1}} \frac{1}{z^{2}} D_{f_{1}}^{h}(z) \frac{d \hat{\sigma}^{A B \rightarrow f_{1} X}}{d p_{T}^{2} d y_{1} d y_{2}},
$$

$d \hat{\sigma}^{A B \rightarrow f_{1} X}$ is the LO pQCD differential cross-section for production of a $f_{1}$ parton pair in a collision of hadrons $A$ and $B$ (nucleons or nuclei), and $D_{f_{1}}^{h}$ is its fragmentation function into the observed hadron, see Ref. [42] for details [80]. Then, we can use Eqs. (15) to compute $\langle\nu\rangle_{p_{h T}, y_{h}}$, $\left\langle Q^{2}\right\rangle_{p_{h T}, y_{h}}$, and $\left\langle z_{h}\right\rangle_{p_{h T}, y_{h}}$. These values parametrize the fixed- $y_{h}$ trajectories $\left\{\left(\langle\nu\rangle_{p_{T}, \bar{y}},\left\langle Q^{2}\right\rangle_{p_{T}, \bar{y}}\right) ; p_{T} \geq p_{0}\right\}$ and $\left\{\left(\langle\nu\rangle_{p_{T}, \bar{y}},\left\langle z_{h}\right\rangle_{p_{T}, \bar{y}}\right) ; p_{T} \geq p_{0}\right\}$ in the DIS-equivalent phase space.

As an example, in Fig. 3 I considered NN collisions at RHIC top energy $\sqrt{s}=200 \mathrm{GeV}$ and at fixed target energies $\sqrt{s}=17-27 \mathrm{GeV}$, and plotted the fixed$y_{h}$ trajectories in the DIS-equivalent phase space. The range of $p_{T}$ spanned along each trajectory is tabulated in Table IV. The spanned range in $Q^{2}$ is limited by the maximum $p_{T}$ at each rapidity, according to Eq. (23). As expected, the larger the rapidity $y_{h} \approx y_{1}$ the smaller the spanned $\nu$. RHIC trajectories with $y_{h} \lesssim-2$ span pretty low values of $\nu \lesssim 60$ and large values of $z_{h} \gtrsim 0.5$, where the EMC and HERMES experiments have shown non negligible cold nuclear matter suppression of hadron production. At higher rapidity, the larger spanned values of $\nu$ will make cold nuclear matter effects less prominent. The consequences of these remarks for the interpretation of hadron production in $h+A$ and $A+A$ collisions will be further discussed in Section IV and V.

\section{FINAL STATE COLD NUCLEAR QUENCHING IN $h+A$ COLLISIONS.}

As we have seen, a parton produced at negative rapidity, $y-y_{c m}<0$, in a $h+A$ collision travels in the 

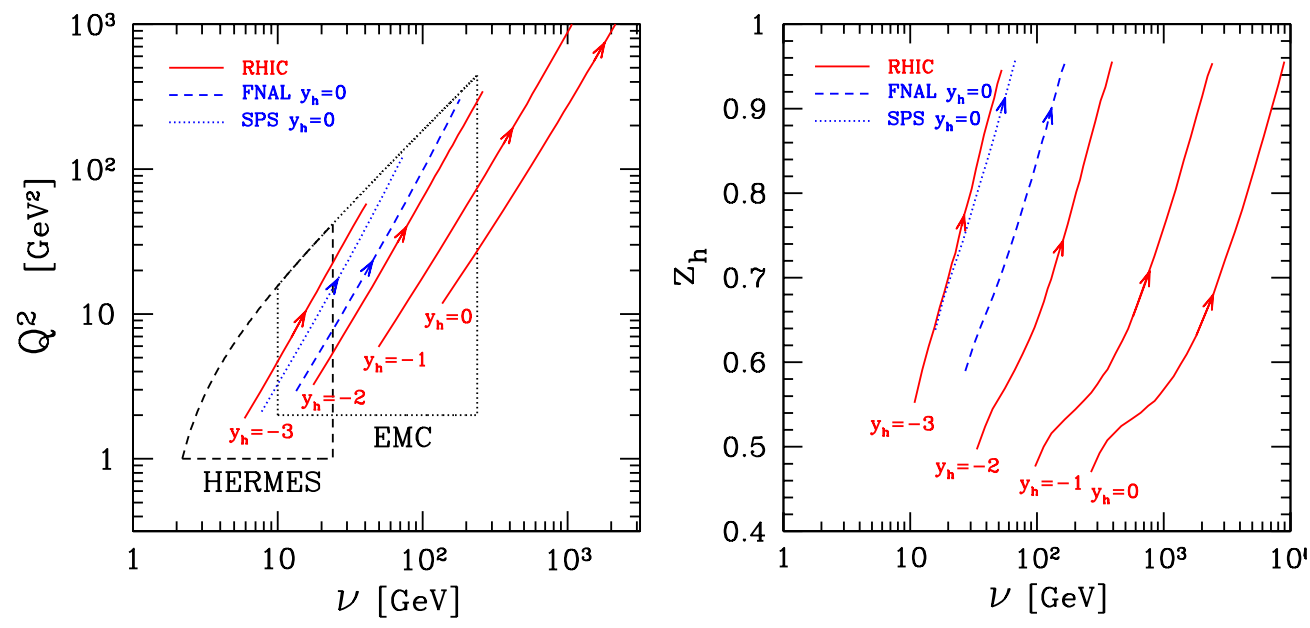

FIG. 3: Right: Fixed- $y_{1}$ NN trajectories plotted in the DIS-equivalent $\left(\nu, Q^{2}\right)$ phase space for $\mathrm{RHIC}$ at $\sqrt{s}=200 \mathrm{GeV}$ and various rapidities, for FNAL and SPS at midrapidity. The dot-dashed line encloses the HERMES phase space; the dashed line encloses the EMC phase space. The arrow indicates the direction of increasing $\left\langle p_{T}\right\rangle$ and $\left\langle z_{h}\right\rangle$. Left: Trajectories in the $\left(\nu, z_{h}\right)$ plane. The arrows indicate increasing $p_{T}$ and $Q_{2}$.

\begin{tabular}{c|c|c|cccc|} 
& SPS & FNAL & \multicolumn{4}{|c|}{ RHIC } \\
& $\sqrt{s}=17.5 \mathrm{GeV}$ & $\sqrt{s}=27.4 \mathrm{GeV}$ & \multicolumn{3}{|c|}{$\sqrt{s}=200$} & $\mathrm{GeV}$ \\
\hline$y_{h}$ & 0 & 0 & 0 & -1 & -2 & -3 \\
$p_{h T}$ & $1-8$ & $1-12$ & $1-90$ & $1-60$ & $1-25$ & $1-9$ \\
\hline
\end{tabular}

TABLE IV: Range of average $\langle p T\rangle$ spanned along RHIC trajectories at fixed rapidity $y_{1}$ and $\sqrt{s}=200 \mathrm{GeV} \cdot p_{h T}$ is quoted in $\mathrm{GeV}$.

same direction as the target nucleus: seen in the nucleus rest frame, it appears to move slowly and corresponds to a low value of $\nu$ in the language of $\ell+A$ collisions. Therefore, based on the observed suppression of hadron production in lepton-nucleus DIS [9-12, 12] at low $\nu$, and on the kinematic analogy between DIS and NN collisions discussed in the previous sections, we can expect nonnegligible hadron suppression due to FS interactions in cold nuclear matter also in $h+A$ and $A+A$ collisions.

Discussion of medium effects is best carried out in the medium rest frame: in the case of cold nuclear matter in $\ell+A$ and $h+A$ collisions it is the nucleus rest frame. I am interested here in processes characterized by large values of $x_{B} \equiv x_{2} \gtrsim 0.1$, typical of semi-inclusive nDIS measurements at HERMES and large $p_{T}$ hadron production at not too forward rapidity in $h+A$ collisions. In this regime, the hard interaction is well localized inside the nucleus, and the nucleons act incoherently as targets [55]. The process evolves in time as follows, see Fig. 4. First the electron (or a parton belonging to the proton) penetrates the nucleus, and undergoes a localized hard collisions. Then, a "final-state" system of 1 electron and 1 parton (or 2 partons) is produced, with both particles essentially traveling along the beam direction, even for rapidity values far from the center of mass rapidity in the target hemisphere $\left(y-y_{c m}<0\right)$. Later on the final state
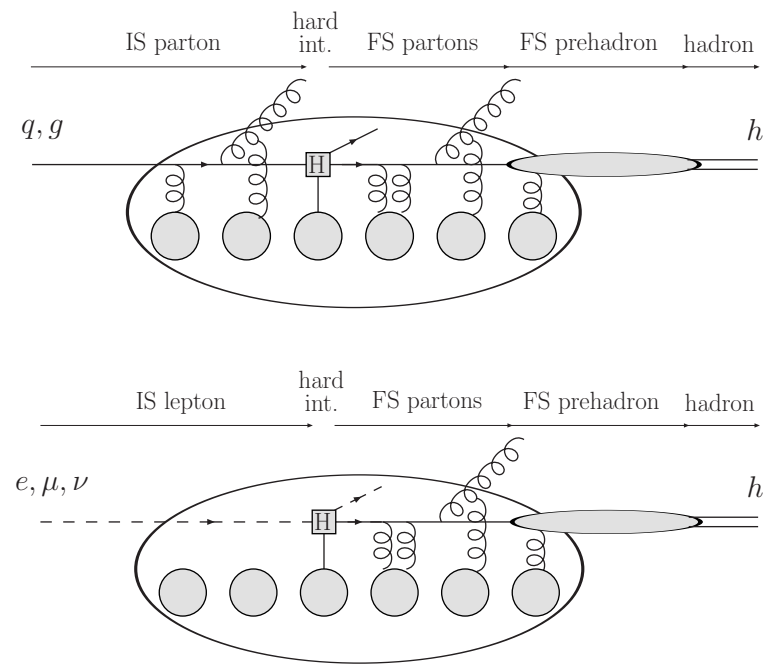

FIG. 4: Top: Initial and final state interactions in $h+A$ collisions in the nucleus rest frame. Bottom: absence of initial state interactions in $\ell+A$ collisions. The nucleus is drawn as an oblong oval for convenience only.

partons hadronize and one of the produced hadrons is detected. The time scale on which hadronization starts after the hard interaction is not at present well known [18]; it may be as small as the nuclear radius [13, 56, 57], in which case the hadronization process would start in the medium. Nuclear medium effects may be classified as initial state (IS) effects on particles before the hard interactions, or final state (FS) effects on particles created after the hard interaction. In the case of $\ell+A$ collisions, electromagnetic reinteractions of the incoming or outgoing lepton are suppressed compared to the strong FS reinteraction of the parton and hadronizing system. 
For $h+A$ collisions one needs in principle to account for both IS and FS interactions [44].

Except at very forward rapidity, I will assume FS and IS effects in $h+A$ collisions to be factorizable because of the large rapidity difference between the IS and FS partons induced by the hard scattering. Then, I will explore the possible size of FS effects on single inclusive hadron production. Differently from $\ell+A$ collisions, the proton projectile interacts with the nucleons along its trajectory. The hard parton produced in the hard collision starts propagating at nearly the speed of light in the same direction but slightly behind the projectile proton. The time scale for parton production, $t_{\text {hard }} \propto 1 / Q$ is much smaller than the time scale for soft particle production in proton-nucleon collisions, $t_{\text {soft }} \propto 1 / \Lambda_{Q C D}$. Hence, we may assume the nuclear medium traversed by the produced parton in $h+A$ collisions to have approximately the same properties as the cold nuclear matter which would be traversed in $\ell+A$ collisions, i.e., the target nucleus itself. With this in mind, we may assume final state hadron quenching effects to be comparable in the 2 cases.

\section{A. Lessons from $\ell+A$ data}

Nuclear modifications of hadron production in $\ell+A$ collisions are typically studied in terms of the hadron multiplicity ratio

$R_{M}^{h}\left(z_{h}, n u, Q^{2}\right)=\frac{1}{N_{A}^{D I S}} \frac{d N_{A}^{h}}{d z_{h} d \nu d Q^{2}} / \frac{1}{N_{D}^{D I S}} \frac{d N_{D}^{h}}{d z_{h} d \nu d Q^{2}}$,

i.e., the single hadron multiplicity on a target of mass number $A$ normalized to the multiplicity on a deuteron target. Then, we can use the dictionary (17) and plot $R_{M}^{h}$ measured in $\ell+A$ collisions as a function of the kinematic variables $p_{T}, y_{1}$ and $z$. This will give a rough estimate of final state effects in $h+A$ collisions. The results are presented in Fig. 5, and the procedure used is discussed below.

Data on $R_{M}$ are usually binned in either $z_{h}, \nu$ or $Q^{2}$. Except for the EMC data, they are presented alongside the average value of the unbinned variables. For HERMES data [11-13], I used the experimentally measured values of the DIS variables to compute the equivalent $p_{T}, y_{1}$ and $z$. For EMC data [10], I used a computation of the average unbinned variables from the GiBUU Monte Carlo generator [58, 59], which was shown to well reproduce the corresponding measurements at HERMES [60]. Another complication arises from the fact that EMC data have been obtained by averaging measurements at 3 electron beam energies, $E_{e}^{\operatorname{trf}}=100 \mathrm{GeV}, 200 \mathrm{GeV}$ and $280 \mathrm{GeV}$; however, the details of such averaging are not immediately clear from the original paper. Therefore, I used the minimum and maximum of the computed average variables to obtain the corresponding minimum and maximum of the NN variables, considered as error band around their average value. Data have been selected to fall into 3 bins in $y_{1}\left(-3.0 \leq y_{1} \leq-2.75\right.$, $-2.5 \leq y_{1} \leq-2.0$, and $\left.-1.5 \leq y_{1} \leq-1.1\right)$, and 1 bin in $z(0.3 \leq z \leq 0.5)$. The choice of $y_{1}$ bins has been made in order to minimize the spread of $y_{1}$ and $z$ inside the bin, and to keep it as much as possible balanced around the middle value. The chosen $z$ bin is the richest in measured data. Furthermore, data with similar $p_{T}$ and from the same target have been combined, with an error band in both $p_{T}$ and $R_{M}$ corresponding to the highest data plus error value and lowest data minus error value, the central value being placed in the middle. Of course, this procedure is a poor man's substitute for direct experimental binning in $p_{T}$ and $y_{1}$.

The results of Fig. 5 clearly show the evolution of final state cold nuclear quenching with rapidity: the quenching increases with decreasing $y_{1}$. This was expected from the kinematic analysis of Section II, which shows a decreasing $\nu$ with decreasing rapidity. The size of hadron quenching is not small, especially for large nuclei and small $y_{1}$ rapidity. Its evolution with $z$ is not shown in the figure because of large overlapping error bars arising in the rebinning procedure. However, the original HERMES and EMC $z_{h}$-distributions clearly show an increasing quenching with increasing $z_{h}$, especially at large $z_{h} \gtrsim 0.5$, where most of hadron production in $h+A$ collisions takes place. Note also that quenching increases with the target atomic number.

As evident from Fig. 5, the $p_{T}$ range covered by HERMES and EMC is quite limited compared to the $p_{T}$ for which hadron production in $h+A$ and $A+A$ can be measured. As remarked in Section III A this situation can be improved with a rebinning of EMC data, or with new measurements of hadron attenuation in $\mu+A$ collisions at the COMPASS experiment, which can in principle reach up to $p_{T} \approx 8-10 \mathrm{GeV}$.

\section{B. Theoretical estimate for $h+A$ collisions}

As already remarked, in DIS, one has experimental control over all the kinematic variables. In $\mathrm{h}+\mathrm{A}$ collisions $Q^{2}$ and, most importantly for our considerations, $z$ are not experimentally accessible. The non-trivial correlation of these variables with the measurable ones is clearly seen in Fig. 3. Moreover, the dependence of hadron quenching on the target atomic number $A$ does not seem to follow any simple law [13, 58, 61]. For these reasons, it is not possible to directly use the results of Fig. 5 to estimate cold nuclear matter effects in $\mathrm{h}+\mathrm{A}$ collisions, but we need to resort to model computations. There exist 2 classes of models which can reproduce nDIS data: (i) energy loss models [61-66], which assume that partons hadronize well outside the target nucleus, and loose energy because of gluon radiation induced by rescatterings inside the target; (ii) prehadron absorption models [57, 58,60,61, 67-69], which assume 

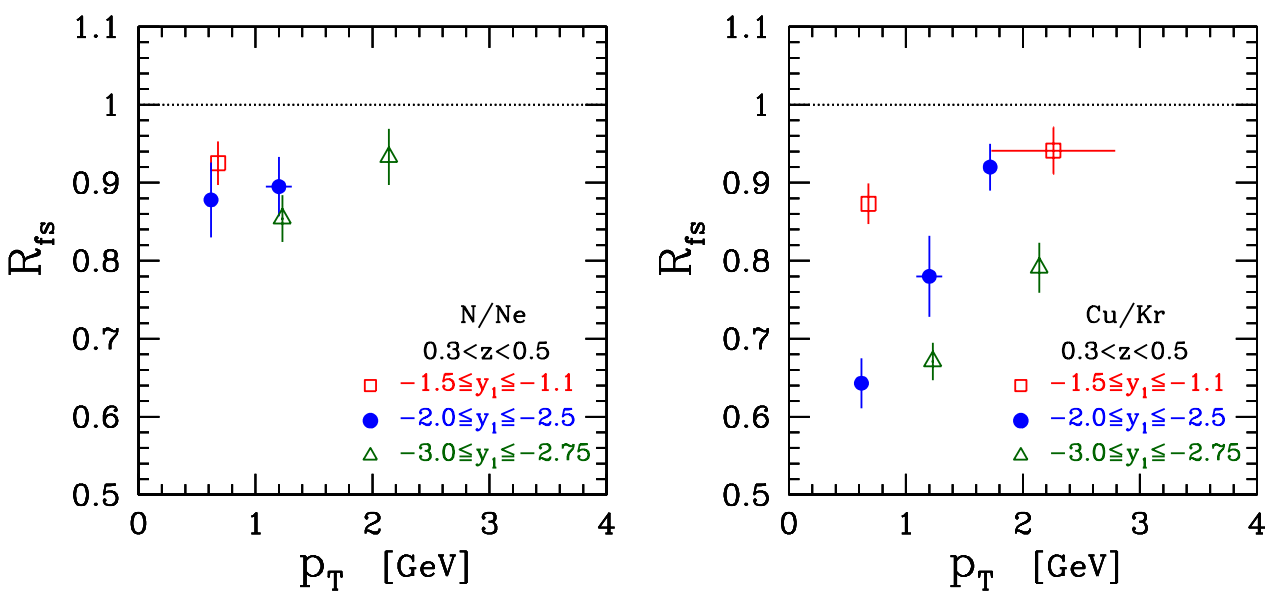

FIG. 5: Cold jet quenching in $\mathrm{d}+\mathrm{A}$ collisions on light and heavy targets at $y_{1} \approx-1.3,-2.25,-3.0$ and $z \approx 0.4$, obtained from HERMES and EMC data on heavy and light targets. See main text for details.

that a colorless prehadron is produced inside the target and can be "absorbed" via inelastic scatterings on the nucleons. As already remarked, the order of magnitude of the parton lifetime has not yet been experimentally or theoretically established, and both classes of models remain viable [18]. Hadron production in $h+A$ collisions has a large contribution from gluon fragmentation, but this process has not been incorporated in absorption models, so far. Therefore I chose to use energy loss models for our estimate. In particular, I will use the BDMS framework as implemented by Salgado and Wiedemann in $[70,71]$ and applied to nDIS in [61, 62]. In this model, the nucleus is considered at rest. A parton, created with energy $E \approx \nu$ in the hard interaction, travels through the nucleus and experiences multiple scatterings and induced gluon bremsstrahlung. Hence, it starts the hadronization process with a reduced energy $E-\Delta E$ where $\Delta E$ is the energy of the radiated gluons. The reduced quark energy at the time of hadronization is translated into a shift of $z$ in the vacuum fragmentation function $D$ [72]. The medium modified FF is then computed as

$$
\begin{aligned}
\tilde{D}_{f / A}^{h}\left(z, Q^{2}, E, E_{h} ; \vec{r}\right) & =\int_{0}^{E_{q}} d \Delta E p\left(\Delta E ; \bar{\omega}_{c}, \bar{R}\right) \\
& \times \frac{1}{1-\Delta E / E} D_{f}^{h}\left(\frac{z}{1-\Delta E / E}, Q^{2}\right)+p_{0}(\bar{R}) D_{f}^{h}\left(z, Q^{2}\right),
\end{aligned}
$$

where the quenching weight $\mathcal{P}(\Delta E)=p(\Delta E)+p_{0} \delta(\Delta E)$ [71] is the probability distribution of an energy loss $\Delta E$, with $p(\Delta E)$ its continuous part and $p_{0}$ the probability of no energy loss. The quenching weight is computed for a static and uniform medium with characteristic gluon energy $\omega_{c}=0.5 \hat{q} L^{2}$ and size parameter $R=\omega_{c} L$, with $L$ the medium length and $\hat{q}$ the transport coefficient of the medium, which characterizes the average transverse momentum squared gained by the parton per unit inmedium path-length [73, 74]. However, the nucleus den- sity is static but non-uniform, hence the dependence of $\tilde{D}$ on the parton production point $\vec{r}$, which on the r.h.s. is implicit in the definition of suitable static-equivalent $\bar{\omega}_{c}$ and $\bar{R}$ [70], see Eqs. (32)-(33). They depend on a single parameter, the transport coefficient $\hat{q}_{0}$ at the center of a reference nucleus. The outlined energy-loss model can well describe light hadron suppression in $\ell+A$ collisions at HERMES with $\hat{q}_{0}=0.5 \mathrm{GeV}^{2} / \mathrm{fm}$, fitted to $\pi^{+}$production on $K r$ targets [18, 61]. I will use the same value for computations in $h+A$ collisions.

The mean free path for a parton in the target nucleus is $\lambda=\left(\sigma \rho_{A}(\vec{r})\right)^{-1}$, where $\sigma$ is the partonic cross-section and $\rho_{A}(\vec{r})$ the nuclear density. Assuming $\sigma$ to be independent of the atomic number, I can define a position-dependent transport coefficient,

$$
\hat{q}_{A}(\vec{b}, y)=\frac{\hat{q}_{0}}{\rho_{0}} \rho_{A}(\vec{b}, y)
$$

where $\hat{q}_{0}=\hat{q}_{\bar{A}}(0,0)$ is the transport coefficient at the center of a reference nucleus of atomic number $\bar{A}$, and $\rho_{0}=\rho_{\bar{A}}(0,0)$. Next, consider a parton produced at $\vec{r}=\left(\vec{r}_{T}, r_{3}\right)$ which propagates in the nucleus along the $r_{3}$ direction. Its average path-length $\bar{L}_{A}$ can be defined as

$$
\bar{L}_{A}(\vec{r})=2 \frac{\int_{r_{3}}^{\infty} d s\left(s-r_{3}\right) \rho_{A}\left(\vec{r}_{T}, r_{3}\right)}{\int_{r_{3}}^{\infty} d s \rho_{A}\left(\vec{r}_{T}, r_{3}\right)},
$$

and the average nuclear density $\bar{\rho}_{A}$ seen by the quark as

$$
\bar{\rho}_{A}(\vec{r})=\frac{\int_{r_{3}}^{\infty} d s \rho_{A}\left(\vec{r}_{T}, r_{3}\right)}{\bar{L}_{A}\left(\vec{r}_{T}, r f_{3}\right)}
$$

Then, from Eq. (28), the average transport coefficient experienced by the quark can be defined as

$$
\overline{\hat{q}}_{A}(\vec{r})=\frac{\hat{q}_{0}}{\rho_{0}} \bar{\rho}_{A}(\vec{r}) .
$$


For a uniform hard-sphere of nuclear density $\rho_{A}(\vec{r})=$ $\rho_{0} \theta\left(R_{A}-|\vec{r}|\right)$, the above definitions give $\bar{L}_{A}=R_{A}-r_{3}$, $\bar{\rho}_{A}=\rho_{0}$, and $\overline{\hat{q}}_{A}=\hat{q}_{0}$ as it should be. Finally, the average characteristic gluon energy $\bar{\omega}_{c}$ and size parameter $\bar{R}$ can be defined as follows:

$$
\begin{aligned}
\bar{\omega}_{c}(\vec{r}) & \equiv \frac{1}{2} \overline{\hat{q}}_{A}(\vec{r}) \bar{L}_{A}^{2}(\vec{r})=\int_{r_{3}}^{\infty} d s(s-y) \hat{q}_{A}\left(\vec{r}_{T}, s\right) \\
\bar{R}(\vec{r}) & \equiv \bar{\omega}_{c}(\vec{r}) \bar{L}_{A}(\vec{r})=\frac{2 \bar{\omega}_{C}^{2}(\vec{r})}{\int_{r_{3}}^{\infty} d s \hat{q}_{A}\left(\vec{r}_{T}, s\right)},
\end{aligned}
$$

These equations have also been used in Ref. [76, 77] for computations of jet quenching in the hot nuclear medium created in $\mathrm{A}+\mathrm{A}$ collisions. Note that they depend on only one parameter, $\hat{q}_{0}$. We can also see that

$$
\overline{\hat{q}}_{A}(\vec{r})=\frac{2}{\bar{L}_{A}^{2}(\vec{r})} \int_{r_{3}}^{\infty} d s\left(s-r_{3}\right) \hat{q}_{A}\left(\vec{r}_{T}, s\right),
$$

as in Ref. [70]. In that paper it was proven that one can approximate the quenching weight for a dynamically expanding medium with the quenching weight for an equivalent static (and uniform) medium characterized by the average $\overline{\hat{q}}_{A}$. However, the natural parameters of the quenching weight are the gluon characteristic energy and the size parameter. Hence, the scaling law is more properly expressed by saying that the equivalent static and uniform medium is characterized by the average $\bar{\omega}_{c}$ and $\bar{R}$ [77]. For a parton propagating in a static but non-uniform medium, as in our case, the spatial non-uniformity is equivalent to a time evolution of the medium. Therefore, as a rough ansatz, we may generalize the SW scaling law to the case of the static but non-uniform medium encountered in nDIS, and use Eqs. (32)-(33) in the quenching weight evaluation. Note, however, that the suitability of a single parameter $\hat{q}$ to describe cold nuclear matter has been recently questioned in Ref. [44].

The parton production cross-section can be computed in LO pQCD as discussed in Section III B. Then the hadron production cross-section including cold nuclear jet quenching can be written as

$$
\begin{aligned}
\frac{d \sigma^{p A \rightarrow h X}}{d p_{T}^{2} d y_{1} d y_{2}} & =\int \frac{d z}{z^{2}} d y_{1} d y_{2} \\
& \times \sum_{f_{1}} \frac{d \hat{\sigma}^{p p \rightarrow f_{1} X}}{d p_{T}^{2} d y_{1} d y_{2}} \tilde{D}_{f_{1} / A}^{h}\left(z, Q^{2}, E, E_{h} ; \vec{r}\right)
\end{aligned}
$$

where up to terms of order $O\left(M^{2} / s\right)$, the target rest frame parton and hadron energy are

$$
E=p_{T} \cosh \left(y_{1}^{*}\right) \quad E_{h}=m_{h T} \cosh \left(y_{h}^{*}\right),
$$

where $y_{i}^{*}=y_{i}+\log (\sqrt{s} / M)$ are the target rest frame rapidities of the parton and the hadron. Isospin corrections related to the target nucleus have been included in the partonic cross section $d \hat{\sigma}^{p p \rightarrow f_{1} X}$. Finally, we can quantify cold matter final state energy loss effects by the ratio of the above discussed cross section for collisions on 2 targets of atomic number $A$ and $B$ :

$$
R_{f s}^{h}\left(p_{T}, \bar{y}\right)=\frac{d \sigma^{p A \rightarrow h X}}{d p_{T}^{2} d y_{1} d y_{2}}\left[\frac{d \sigma^{p B \rightarrow h X}}{d p_{T}^{2} d y_{1} d y_{2}}\right]^{-1}
$$

and the amount of hadron quenching by $1-R_{f s}^{h}$.

The computed $R_{f s}^{h}$ for charged pion production with no centrality selection is presented in Fig. 6. When examining these plots, one should keep in mind that they are intended only o show the extent of the suppression effects on hadron production due to cold nuclear matter energy loss of the final state parton. They do not include the related transverse momentum broadening nor initial state effects, which will be commented on in the next section. The plots show a substantial final state hadron quenching already for midrapidity hadrons at SPS and FNAL energy, and for $y_{h}<-2$ at RHIC. The quenching at RHIC is reduced when increasing the rapidity, but is still non-negligible at $y_{h}=0$, where it is of order $5 \%$ at $p_{T} \gtrsim 10 \mathrm{GeV}$. This may explain the small $\pi^{0}$ quenching apparent in recent midrapidity PHENIX data [41, 43]. Final state cold quenching at RHIC should then quickly disappear at forward rapidity. At the LHC, we may expect negligible final state effects at $y_{h} \gtrsim 3$ because of the rapidity shift $\Delta y$ in Table III. I also found a small hadron flavor dependence at small $p_{h T}$, not shown in the plots, showing less suppression for kaon and proton production than for pion production. It would be interesting to compare these estimates, obtained in the quenching weight formalism of Salgado and Wiedemann [71], with the results of other energy loss implementations such as the twist-4 formalism of Refs. [64-66] and the reaction operator approach of Ref. [44]. A nice comparison of the available formalisms has been recently discussed in Ref. [78].

\section{INITIAL VS. FINAL STATE EFFECTS}

Before discussing the phenomenological relevance of the estimate of cold nuclear matter effects obtained in the last section, we need to discuss the importance of initial state effects, so far neglected.

The initial state parton suffers multiple scatterings and medium-induced gluon radiation. In a simple phenomenological model [33], the resulting energy loss may be accounted for by a shift of the incoming parton fractional momentum, $x_{1} \rightarrow x_{1}(1-\epsilon)$, with $\epsilon=\kappa A^{1 / 3}$ the fractional IS energy loss. The effect of such energy loss is felt in a kinematic region where the flux of incoming partons varies rapidly with $x_{1}$, typically at large rapidity. Numerical estimates from [33] indicate that IS state energy loss in $d+A u$ collisions at $\sqrt{s}=19.4$ become relevant only at forward rapidity $y-y_{c m} \gtrsim 0$. According to the rapidity shifts listed in Table III, we may expect a similar conclusion to hold for $y-y_{c m} \gtrsim 2(5)$ at RHIC (LHC). 

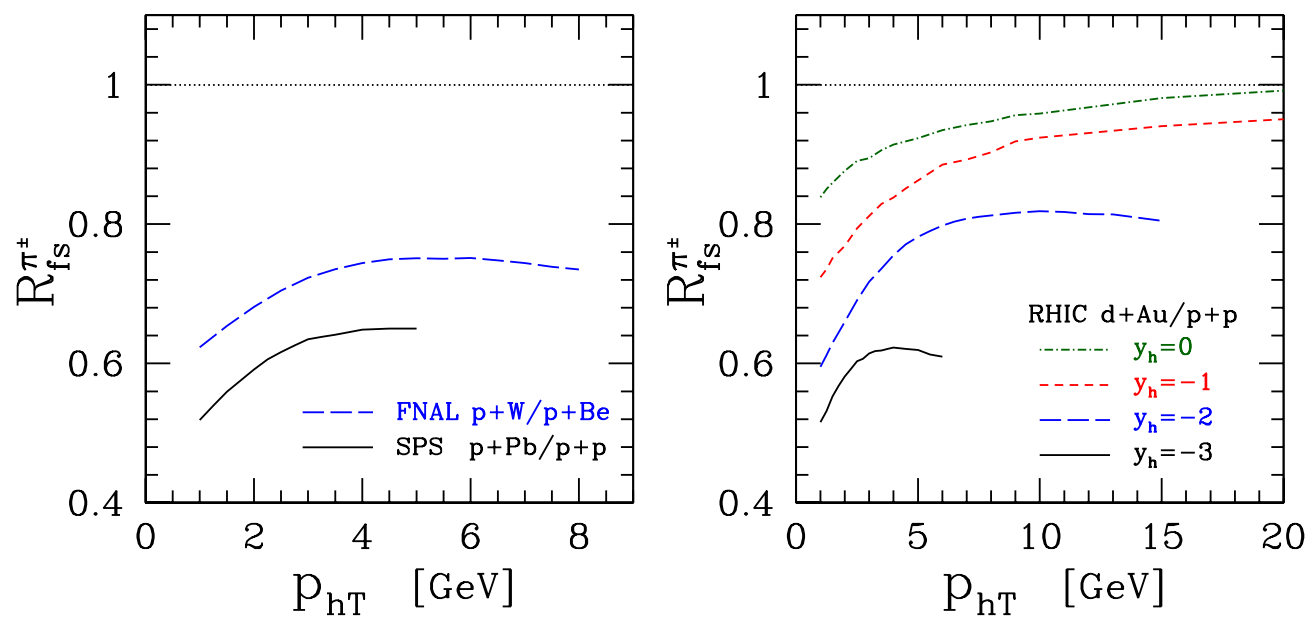

FIG. 6: Energy loss model estimate of final state hadron quenching in cold nuclear matter for midrapidity pions at SPS and FNAL, and several negative rapidities at RHIC.

If the final state parton is long lived, as assumed in the theoretical estimates of the previous section, the medium affects hadron production mainly through elastic and radiative energy losses. In this case, the FS energy loss enters the computations as a shift in $z$ of the fragmentation function, see Eq. (27). Hence, differently from IS energy loss, it is large in regions where the fragmentation functions changes rapidly in $z$, namely at large $z$. At fixed $p_{h T}$ the average $\langle z\rangle$ increases with decreasing rapidity and decreasing $\sqrt{s}$ (see Fig. 7 right). Coupling this with a decrease in final state parton energy $E_{f}$ with decreasing rapidity, it is easy to explain the behavior and large size of final state suppression shown in Fig. 6.

A consistent framework for considering the interplay of IS and FS energy loss in the reaction operator formalism is discussed in Ref. [44], which presents numerical results for the partonic fractional energy loss $\Delta E / E$ in a case study of a homogeneous medium of fixed length $L=5 \mathrm{fm}$. At any given parton energy $E$, the FS fractional energy loss is generally smaller than the IS fractional energy loss. They both start at around $10 \%$ when $E=10 \mathrm{GeV}$, but FS energy loss tends rapidly to 0 as $E$ increases, contrary to IS energy loss which stabilizes around $5 \%$ at $E \gtrsim 1$ $\mathrm{TeV}$. However, particle production at fixed rapidity in $h+$ $A$ and $A+A$ collisions shows a strong correlation between the IS parton energy $E_{i}$ and the FS parton energy $E_{f}$, see Fig. 7 left. As a result, for midrapidty hadrons at SPS we have comparable IS and FS state energy loss of $5-10 \%$. For midrapidity hadrons at RHIC, FS energy loss becomes quite small, and IS radiation is about $5 \%$. For backward rapidity production, $y_{h}-y_{c m}=-3$, FS energy loss is now larger than IS energy loss, viz., $10 \%$ vs. $5 \%$. A detailed computation including realistic nuclear geometry is needed to quantify their effect on hadron spectra.

In summary, IS and FS cold nuclear matter effects are expected to be dominant in different rapidity regions, viz., at forward and backward rapidity, where the estimates presented in this paper and in Ref. [33] indicate that they are large. Their effect on the midrapidity region has to be more carefully and quantitatively considered: it depends on the center of mass energy of the collision, and can be expected to decrease with increasing $\sqrt{s}$.

\section{DISCUSSION AND CONCLUSIONS}

In this paper, I have examined the role of final state interactions in cold nuclear matter in modifying hadron production on nuclear targets with leptonic or hadronic beams. Initial state parton energy loss has been considered in $[22,23,33]$. Since in $\ell+A$ collisions only FS interactions are present, I built a kinematic dictionary that relates the variables used for the discussion of nDIS and heavy-ion $A+B$ collisions, and demonstrated the (limited) extent to which available experimental data on hadron suppression in nDIS can give direct information on final state cold nuclear matter effects in $A+B$ collisions. In this respect, the EIC $[50,51]$ will be able to efficiently cover the regions in phase space which the HERMES [13] and EMC [10] experiments could not examine. A nearly full coverage of the SPS and FNAL phase space may alternatively be achieved either by a reanalysis of EMC data, or by new measurements of hadron attenuation at the COMPASS experiment [52]. The latter option is particularly interesting: COMPASS has a similar kinematic coverage to EMC, but higher luminosity and very good particle identification capabilities. Therefore a $\mu+A$ program at COMPASS, building on the knowledge accumulated at the HERMES [13] and CLAS experiments $[14,79]$, would greatly improve our knowledge of the space-time evolution of hadronization, and gather vital data for the interpretation of $h+A$ and $A+A$ collisions and the quest for the Quark Gluon Plasma. 

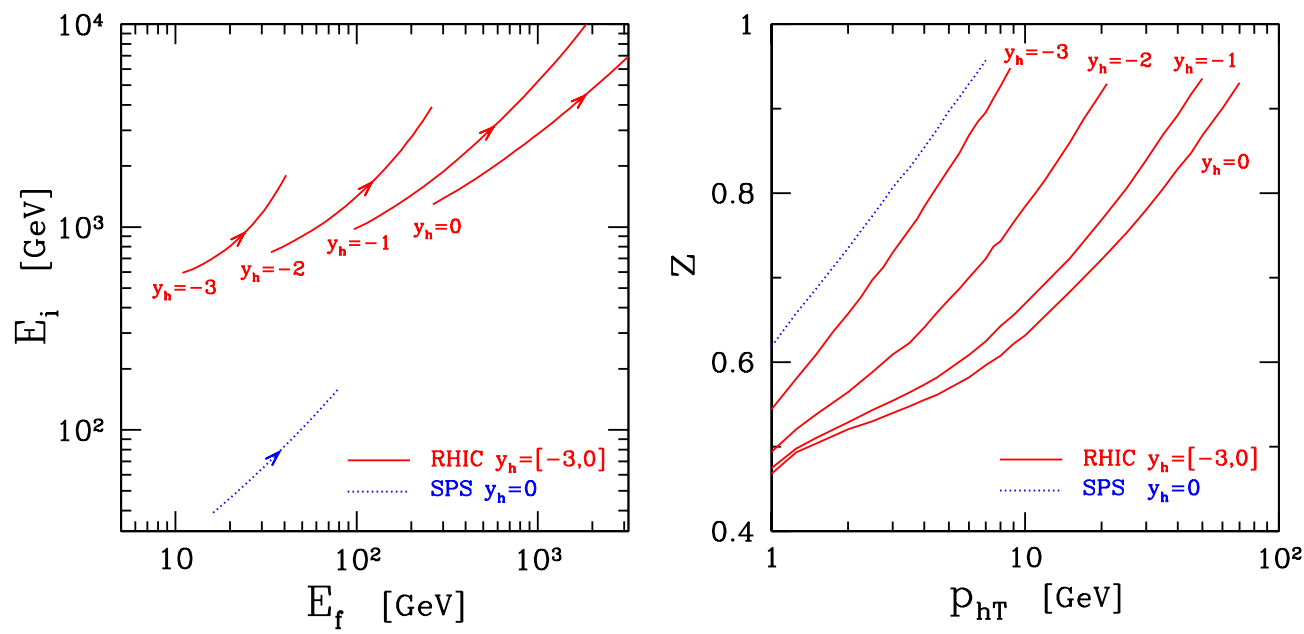

FIG. 7: Correlation of initial and final state parton energy (left), and hadron transverse momentum and fractional momentum (right).

Hadron production in $h+A$ and $A+A$ collisions is affected by cold nuclear matter in 2 ways.

1. IS and FS energy loss, and possibly FS prehadron absorption, suppress hadron spectra by non negligible amounts at forward [33] and backward rapidity, respectively.

2. The transverse momentum broadening associated with induced radiation and multiple scatterings in the medium will modify the hadron $p_{h T}$ spectrum, further suppressing it at $p_{T} \lesssim 1-2 \mathrm{GeV}$ and enhancing it at intermediate momenta up to $p_{T} \approx 5-6 \mathrm{GeV}[27]$.

I used an energy loss model based on the BDMS formalism and tuned to $\ell+A$ data, to estimate the size of final state cold hadron quenching in hadronic collisions, which was found to be large at midrapidity at fixed target SPS and FNAL energy, and at backward rapidity at RHIC energy. It will be interesting to compare this result with estimates based on the GLV [44] and high-twist [64$66]$ formalism for energy loss, and on nuclear absorption models [57, 58, 67].

In this paper, only the induced FS suppression has been discussed and estimated. However, for phenomenological applications to $h+A$ and $A+A$ collisions the interplay of suppression and enhancement of hadron spectra will need to be elucidated. In $h+A$ collisions, cold nuclear effects will be important to understand the evolution with rapidity of the nuclear modification factors, which decreases at forward rapidity [15-17] but increases at negative rapidity [28-31]. In $A+A$ collisions, the time scale for the formation and equilibration of the QuarkGluon Plasma is much larger than the time scale for cold nuclear matter interactions: $t_{\text {cold }} \ll t_{e q}$. Hence, both cold and hot quenching should be considered for a correct interpretation of experimental data. At RHIC midrapidity, I estimated cold quenching of hadron spectra to be of order $5-10 \%$. It is much smaller than the observed factor 4-5 hot medium quenching observed in central $A u+A u$ collisions, and is negligible in first instance. At LHC cold nuclear matter effects are likely to be negligible in a large midrapidity interval $|y| \lesssim 3$. At SPS energy, where cold nuclear matter effects may be of the same order of magnitude or larger than hot medium effects, they both need to be taken into account in any QCD tomographic analysis to detect and extract the properties of the Quark Gluon Plasma.

\section{Acknowledgments}

I am grateful to M. Djordjevic, U. Heinz, J.W. Qiu, M. Rosati, G. Sterman and I. Vitev for valuable discussions. I would also like to thank P. di Nezza for a careful reading of the manuscript and for drawing my attention to the possibilities offered by the COMPASS experiment, and K. Gallmesiter for providing me with GiBUU Monte Carlo computations of average kinematic variables in nDIS. This work is partially funded by the US Department of Energy grant DE-FG02-87ER40371.
[1] I. Arsene et al. [BRAHMS], Nucl. Phys. A 757 (2005) 1.

[2] B. B. Back et al., Nucl. Phys. A 757, 28 (2005).

[3] J. Adams et al. [STAR], Nucl. Phys. A 757, 102 (2005).
[4] K. Adcox et al. [PHENIX], Nucl. Phys. A 757, 184 (2005)

[5] M. Gyulassy, I. Vitev, X. N. Wang and B. W. Zhang, in "Quark Gluon Plasma 3", R.C. Hwa and X.N. Wang eds., 
World Scientific, Singapore [arXiv:nucl-th/0302077].

[6] A. Kovner and U. A. Wiedemann, in "Quark Gluon Plasma 3", R.C. Hwa and X.N. Wang eds., World Scientific, Singapore [arXiv:hep-ph/0304151.]

[7] I. Vitev, J. Phys. G 30 (2004) S791.

[8] M. Gyulassy and L. McLerran, Nucl. Phys. A 750 (2005) 30.

[9] L. S. Osborne et al., Phys. Rev. Lett. 40 (1978) 1624.

[10] J. Ashman et al. [EMC] Z. Phys. C 52 (1991) 1.

[11] A. Airapetian et al. [HERMES], Eur. Phys. J. C 20 (2001) 479.

[12] A. Airapetian et al. [HERMES], Phys. Lett. B 577 (2003) 37.

[13] A. Airapetian et al. [HERMES Collaboration], arXiv:0704.3270 [hep-ex].

[14] K. Hafidi [CLAS], AIP Conf. Proc. 870 (2006) 669.

[15] I. Arsene et al. [BRAHMS], Phys. Rev. Lett. 93 (2004) 242303.

[16] J. Adams et al. [STAR], Phys. Rev. Lett. 97 (2006) 152302

[17] B. B. Back et al. [PHOBOS Collaboration], Phys. Rev. C 70 (2004) 061901.

[18] A. Accardi, Eur. Phys. J. C 49 (2007) 347.

[19] J. Badier et al. [NA3], Phys. Lett. B 104 (1981) 335.

[20] D. M. Alde et al. [FNAL E772], Phys. Rev. Lett. 64 (1990) 2479.

[21] M. A. Vasilev et al. [FNAL E866] Phys. Rev. Lett. 83 (1999) 2304.

[22] M. B. Johnson et al., Phys. Rev. C 65 (2002) 025203 [arXiv:hep-ph/0105195].

[23] F. Arleo, Phys. Lett. B 532 (2002) 231 [arXiv:hep$\mathrm{ph} / 0201066]$.

[24] M. B. Johnson, B. Z. Kopeliovich, M. J. Leitch, P. L. McGaughey, J. M. Moss, I. K. Potashnikova and I. Schmidt, Phys. Rev. C 75 (2007) 035206.

[25] J. W. Cronin et al. Phys. Rev. D 11 (1975) 3105.

[26] D. Antreasyan et al. Phys. Rev. D 19 (1979) 764.

[27] A. Accardi, arXiv:hep-ph/0212148.

[28] J. Adams et al. [STAR], Phys. Rev. C 70 (2004) 064907.

[29] S. S. Adler et al. [PHENIX], Phys. Rev. Lett. 94 (2005) 082302 [arXiv:nucl-ex/0411054].

[30] B. I. Abelev et al. [STAR], arXiv:nucl-ex/0609021.

[31] B. I. Abelev et al. [STAR], arXiv:nucl-ex/0703016.

[32] T. Alber et al. [NA35], Eur. Phys. J. C 2 (1998) 643 [arXiv:hep-ex/9711001].

[33] I. Vitev, T. Goldman, M. B. Johnson and J. W. Qiu, Phys. Rev. D 74(2006) 054010.

[34] D. Kharzeev, Y. V. Kovchegov and K. Tuchin, Phys. Lett. B 599 (2004) 23 [arXiv:hep-ph/0405045].

[35] J. Jalilian-Marian and Y. V. Kovchegov, Prog. Part. Nucl. Phys. 56 (2006) 104 [arXiv:hep-ph/0505052].

[36] R. Vogt, arXiv:hep-ph/0405060.

[37] B. Z. Kopeliovich, J. Nemchik, I. K. Potashnikova, M. B. Johnson and I. Schmidt, Phys. Rev. C 72 (2005) 054606.

[38] R. C. Hwa, C. B. Yang and R. J. Fries, Phys. Rev. C 71 (2005) 024902 [arXiv:nucl-th/0410111].

[39] A. Accardi, Acta Phys. Hung. A 22 (2005) 289.

[40] G. G. Barnafoldi, P. Levai, G. Papp and G. Fai, Nucl. Phys. A 749 (2005) 291.

[41] S. S. Adler et al. [PHENIX], Phys. Rev. Lett. 98 (2007) 172302 [arXiv:nucl-ex/0610036].

[42] K. J. Eskola and H. Honkanen, Nucl. Phys. A 713 (2003) 167.
[43] B. A. Cole, G. G. Barnafoldi, P. Levai, G. Papp and G. Fai, arXiv:hep-ph/0702101.

[44] I. Vitev, Phys. Rev. C 75 (2007) 064906.

[45] R. D. Field, "APPLICATIONS OF PERTURBATIVE QCD," Addison-Wesley (1989) (Frontiers in physics, 77)

[46] J. C. Collins and D. E. Soper, Nucl. Phys. B 193 (1981) 381 [Erratum-ibid. B 213 (1983) 545].

[47] B. A. Kniehl, G. Kramer and B. Potter, Nucl. Phys. B 582 (2000) 514.

[48] S. Kretzer, Phys. Rev. D 62 (2000) 054001.

[49] P. B. van der Nat [HERMES Collaboration], Acta Phys. Polon. B 35 (2004) 139.

[50] A. Deshpande, R. Milner, R. Venugopalan and W. Vogelsang, Ann. Rev. Nucl. Part. Sci. 55 (2005) 165.

[51] C. Aidala et al. [EIC] with A. Accardi, V. Guzey, T. Lappi, C. Marquet, J. W. Qiu, "Physics Opportunities with $\mathrm{e}+\mathrm{A}$ Collisions at an Electron Ion Collider", available at http://www.phenix.bnl.gov/ $\sim$ dave/eic/PositionPaper_eA.pdf

[52] P. Abbon et al. [COMPASS], arXiv:hep-ex/0703049.

[53] A. Accardi and M. Gyulassy, Phys. Lett. B 586 (2004) 244.

[54] V. Guzey, M. Strikman and W. Vogelsang, Phys. Lett. B 603 (2004) 173.

[55] P. Hoyer, arXiv:hep-ph/9510394.

[56] A. Accardi, arXiv:nucl-th/0604041.

[57] B. Z. Kopeliovich, J. Nemchik, E. Predazzi and A. Hayashigaki, Nucl. Phys. A 740 (2004) 211.

[58] K. Gallmeister and U. Mosel, arXiv:nucl-th/0701064.

[59] K. Gallmeister, private communication.

[60] T. Falter, W. Cassing, K. Gallmeister and U. Mosel, Phys. Rev. C 70 (2004) 054609.

[61] A. Accardi, Acta Phys. Hung. A 27 (2006) 189.

[62] F. Arleo, Eur. Phys. J. C 30 (2003) 213.

[63] F. Arleo, JHEP 0211 (2002) 044

[64] E. Wang and X. N. Wang, Phys. Rev. Lett. 89 (2002) 162301.

[65] X. N. Wang and X. f. Guo, Nucl. Phys. A 696 (2001) 788.

[66] X. f. Guo and X. N. Wang, Phys. Rev. Lett. 85 (2000) 3591.

[67] A. Accardi, D. Grunewald, V. Muccifora and H. J. Pirner, Nucl. Phys. A 761 (2005) 67.

[68] A. Accardi, V. Muccifora and H. J. Pirner, Nucl. Phys. A 720 (2003) 131 .

[69] A. Bialas and M. Gyulassy, Nucl. Phys. B 291 (1987) 793.

[70] C. A. Salgado and U. A. Wiedemann, Phys. Rev. Lett. 89 (2002) 092303.

[71] C. A. Salgado and U. A. Wiedemann, Phys. Rev. D 68 (2003) 014008 [arXiv:hep-ph/0302184].

[72] X. N. Wang, Z. Huang and I. Sarcevic, Phys. Rev. Lett. 77 (1996) 231.

[73] R. Baier, Y. L. Dokshitzer, A. H. Mueller, S. Peigne and D. Schiff, Nucl. Phys. B 484 (1997) 265.

[74] F. Arleo, "Estimates for cold nuclear matter transport coefficients", in Ref. [75], page 141

[75] A. Accardi et al., "Hard probes in heavy ion collisions at the LHC: Jet physics," working group report for CERN Yellow Report, arXiv:hep-ph/0310274.

[76] K. J. Eskola, H. Honkanen, C. A. Salgado and U. A. Wiedemann, Nucl. Phys. A 747 (2005) 511.

[77] A. Dainese, C. Loizides and G. Paic, Eur. Phys. J. C 38 (2005) 461. 
[78] A. Majumder, arXiv:nucl-th/0702066.

[79] W. K. Brooks, Fizika B 13 (2004) 321.

[80] The computations presented in this paper, differ from [42] in 2 respects: I defined $z=p_{h}^{+} / f_{1}^{+}$instead of $z=$ $E_{h} / E_{f_{1}}$, and I regularized the pQCD cross-section in the infrared with a shift $p_{T}^{2} \rightarrow p_{T}^{2}+p_{0}^{2}$ instead of using a sharp cutoff $p_{T}>p_{0}$. The difference is mostly seen at small $p_{h T} \lesssim p_{0}$. 